\title{
The role of the mammalian branchpoint sequence in pre-mRNA splicing
}

\author{
Robin Reed and Tom Maniatis \\ Department of Biochemistry and Molecular Biology, Harvard University, Cambridge, Massachusetts 02138 USA
}

\begin{abstract}
We show that base substitutions in the mammalian branchpoint sequence (BPS) YNCUGAC dramatically reduce the efficiency of pre-mRNA splicing in vitro and alter 3' splice-site selection in vivo. Contrary to current dogma that an adenine residue at the appropriate distance from the $3^{\prime}$ splice site is the primary determinant of lariat formation, we find that many mutations in the BPS virtually abolish splicing even though the position of this adenine is unchanged. Comparison of the analogous single-base changes in the mammalian and yeast BPSs revealed similar relative effects on splicing efficiency. However, in contrast to yeast, mammalian branchpoint mutations that decrease splicing efficiency severely do not prevent spliceosome assembly. Thus, mutations in the mammalian BPS appear to uncouple spliceosome assembly from cleavage at the 5' splice site and lariat formation.
\end{abstract}

[Key Words: Spliceosome; splicing mechanisms; lariat formation; splice-site selection]

Received June 28, 1988; revised version accepted August 23, 1988.

Lariat formation during pre-mRNA splicing in higher eukaryotes usually occurs at an adenine residue in a weakly conserved sequence element, YNCUGAC. This element, designated the branchpoint sequence (BPS), is located at a conserved distance from the $3^{\prime}$ splice site of most introns examined (Keller and Noon 1984; Ruskin et al. 1984; Zeitlin and Efstratiadis 1984; Reed and Maniatis 1985). A role for the BPS in splicing was strongly suggested by the observation that a synthetic intron lacking a normal BPS was spliced more efficiently in vivo when a normal BPS was inserted near the $3^{\prime}$ splice site (Rautmann and Breathnach 1985). Moreover, putative BPSs in a number of introns function as the predominant site of lariat formation in vitro (Reed and Maniatis 1985). Finally, a decrease in the efficiency of splicing in vitro was observed with pre-mRNAs lacking a normal BPS (Padgett et al. 1985; Reed and Maniatis 1985; Ruskin et al. 1985) or containing single-base substitutions in the branch-site nucleotide (Ruskin et al. 1985; Hornig et al. 1986; Freyer et al. 1987). In most of these cases, the failure to abolish splicing in vitro was explained by the finding that cryptic branch sites located within the conserved distance from the $3^{\prime}$ splice site are used in the absence of the normal BPS (Padgett et al. 1985; Reed and Maniatis 1985; Ruskin et al. 1985).

The functional role of the BPS was questioned, however, based on the observation that some pre-mRNAs with altered or deleted BPSs are spliced normally in vivo (Rautmann et al. 1984; Wieringa et al. 1984; Padgett et al. 1985; Ruskin et al. 1985). Thus, the cryptic branch sites in these pre-mRNAs appeared to compensate fully for the loss of the normal BPS in vivo. Taken together, these results suggested that the primary requirement for lariat formation is an adenine nucleotide in the proper location (Green 1986; Padgett et al. 1986; Ruskin et al. 1988 .

In contrast to the mammalian BPS, the BPS analog in yeast (the UACUAAC box) is highly conserved and is essential for both spliceosome assembly and splicing (for reviews, see Green 1986; Padgett et al. 1986; Krainer and Maniatis 1988). These observations led to the view that there is a mechanistic difference in the role of the BPS in yeast and higher eukaryotes. Remarkably, however, a direct comparison between the effects of mutations in the yeast and mammalian BPS has not been reported.

In this paper we show that many mutations in the mammalian BPS, including single-base changes, dramatically decrease the efficiency of splicing in vitro and alter $3^{\prime}$ splice-site selection in vivo. Moreover, the effects on splicing efficiency observed with the BPS mutations parallel the effects of the analogous mutations in yeast. Thus, the BPS may, in fact, play a similar role in splicing in yeast and mammals. In spite of these similarities, however, we find that mutations in the mammalian BPS that decrease splicing efficiency severely do not prevent spliceosome assembly, in contrast to yeast.

\section{Results}

To investigate the role of the mammalian BPS in splicing, we used a cis-competition assay similar to that employed previously to study the involvement of exon sequences in splicing (Reed and Maniatis 1986). In this assay, the RNA precursor contains the $5^{\prime}$ splice site of the first intron of the human $\beta$-globin gene and tandem duplications of the $3^{\prime}$ splice site (Fig. 1). A BPS is located in the normal position adjacent to each of the duplicated 


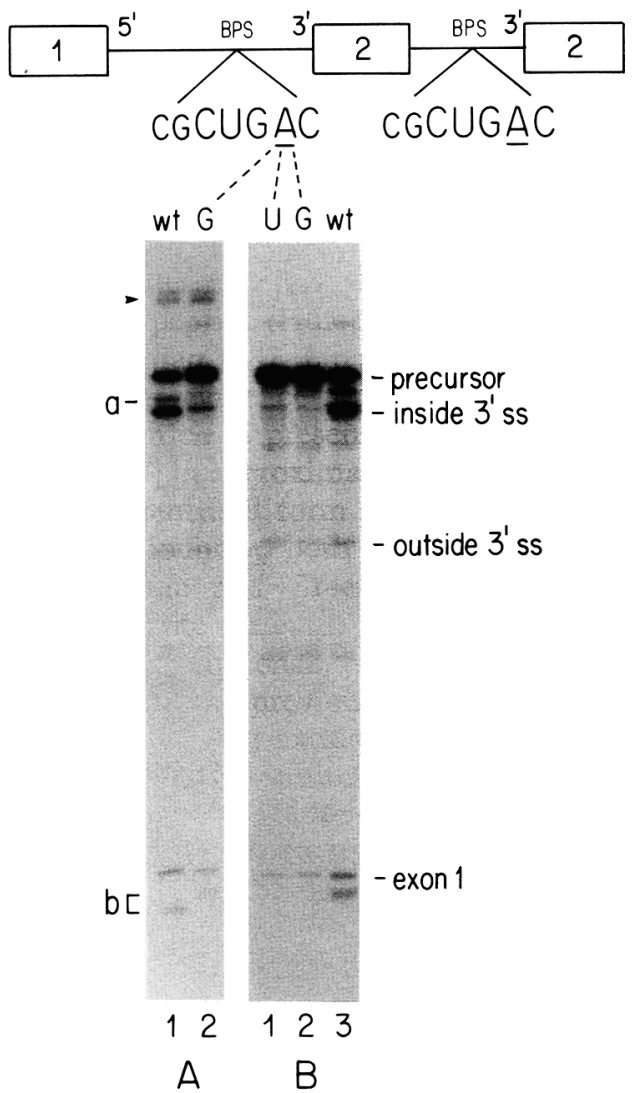

Figure 1. The effect of mutations in the branch-site adenine on splicing in vitro. (Top) The structure of the SP6 RNA precursors spliced in vitro for $1 \mathrm{hr}$ and then analyzed on a $5.5 \%$ denaturing polyacrylamide gel. Boxes indicate exons, lines designate intron sequences. The location of the BPS and 5' and 3' splice sites are shown. The underlined A is the branch site adenine. The nucleotides in the BPS shown in lowercase letters are less conserved then those shown in uppercase letters. $(A)$ Analysis of a BPS mutation in the presence of potential cryptic branch sites. (Lane 1) Normal BPS (CGCUGAC) adjacent to both 3' splice sites; (lane 2) A $\rightarrow$ G change in the BPS adjacent to the internal 3' splice site. (B) Analysis of BPS mutations in the absence of potential cryptic branch sites. (Lane 1) $A \rightarrow U$ change in the BPS adjacent to the internal $3^{\prime}$ splice site; (lane 2) $\mathrm{A} \rightarrow \mathrm{G}$ change in the BPS adjacent to the internal $3^{\prime}$ splice site; (lane 3) normal BPS adjacent to both $3^{\prime}$ splice sites. The precursor RNA and the spliced RNA derived from use of the internal $3^{\prime}$ splice site (inside $3^{\prime}$ ss) and external $3^{\prime}$ splice site (outside $3^{\prime} \mathrm{ss}$ ) are indicated. (a) Lariat-exon intermediate derived from use of the internal $3^{\prime}$ splice site; $(b)$ excised lariat intron derived from use of the internal 3 ' splice site. The faint bands above the precursor are the lariat introns derived from use of the external 3' splice site. The bands marked with an arrowhead in $A$ (lanes 1,2) are present in the unspliced precursor.

$3^{\prime}$ splice sites. In previous studies we found that the internal $3^{\prime}$ splice site is used preferentially in this precursor (Reed and Maniatis 1986). To determine whether alterations in the BPS affect splicing, mutations were introduced into the BPS adjacent to the internal $3^{\prime}$ splice site and the normal BPS was adjacent to the external $3^{\prime}$ splice site. Based on our earlier work, BPS mutations that decrease the efficiency of splicing to the internal $3^{\prime}$ splice site should lead to an increase in the use of the external 3' splice site (Reed and Maniatis 1986). In addition, potential cryptic branch sites were eliminated from both of the duplicated introns to increase the sensitivity of the cis-competition assay. This was achieved by replacing the adenine residues located in the $3^{\prime}$ portion of each intron with other nucleotides, as described in Experimental procedures. A similar strategy, elimination of a potential cryptic branch site, was used in analogous studies of the yeast BPS (Vijayraghavan et al. 1986; Parker et al. 1987).

To establish the feasibility of the cis-competition assay for analysis of BPS mutations and to assess the effect of eliminating the potential cryptic branch sites, we compared the in vitro splicing products generated from precursors containing or lacking these sites. A control precursor containing the normal BPS adjacent to both $3^{\prime}$ splice sites and precursors containing base changes in the branch-site adenine of the internal BPS were examined (Fig. 1). Comparison of the control precursors in the presence (Fig. 1A, lane 1) or absence (Fig. 1B, lane 3) of the potential cryptic branch sites revealed similar splicing patterns. As expected from our previous studies, the internal $3^{\prime}$ splice site is used preferentially. Thus, elimination of the potential cryptic branch sites does not affect splicing of the control precursor significantly.

Substituting the branch-site adenine in the internal BPS with a guanine resulted in a significant decrease in use of the internal $3^{\prime}$ splice site in the presence (Fig. 1A, lane 2) or absence (Fig. 1B, lane 2) of the potential cryptic branch sites. A similar result was obtained when the branchpoint adenine of the internal BPS was changed to $U$ in the precursor lacking the cryptic branch sites (Fig. 1B, lane 1). The decrease in the use of the internal 3' splice site is slightly greater with the precursors lacking the potential cryptic branch sites than with the precursor that retains these sites (Fig. 1). Thus, as expected, the effects of BPS mutations are more severe in the absence of potential cryptic branch sites.

Although BPS mutations decrease the use of the internal 3' splice site, a comparable increase in the use of the external $3^{\prime}$ splice site was not observed (Fig. 1). This result was unexpected based on our previous observation that exon mutations that decrease the efficiency of splicing to the internal $3^{\prime}$ splice site result in normal levels of splicing to the external 3' splice site (Reed and Maniatis 1986). Thus, in contrast to the effect of exon mutations, BPS mutations decrease the overall splicing efficiency of these precursors. A possible reason for this difference, observed with all of the BPS mutants examined, will be discussed below.

We note that the relative levels of splicing to the internal and external 3 ' splice sites with both the mutant and control precursors vary somewhat in different extract preparations, in diluted extracts, and in extracts that have been frozen and thawed (Reed and Maniatis 1986; data not shown). These variations, however, do not affect the interpretation of the results, because splice-site selection in the control and mutant precursors appears to be affected similarly in different extracts. 
The branch-site adenine nucleotide is not sufficient for normal levels of splicing in vitro

To investigate the proposal that the primary determinant of lariat formation is an adenine residue located at the correct distance from the $3^{\prime}$ splice site, we analyzed mutations that maintain the position of the branch-site adenine but alter the surrounding nucleotides in the BPS. The BPS mutations were constructed in the absence of the potential cryptic branch sites, as described in Experimental procedures. Mutants that contain two or more base changes in the BPS but maintain the position of the branch-site adenine (Fig. 2, lanes 2-4) or in which the branch-site adenine is close to the wildtype location (Fig. 2, lane 1) were chosen for analysis using the cis-competition assay. A dramatic reduction in use of the internal $3^{\prime}$ splice site was observed with three
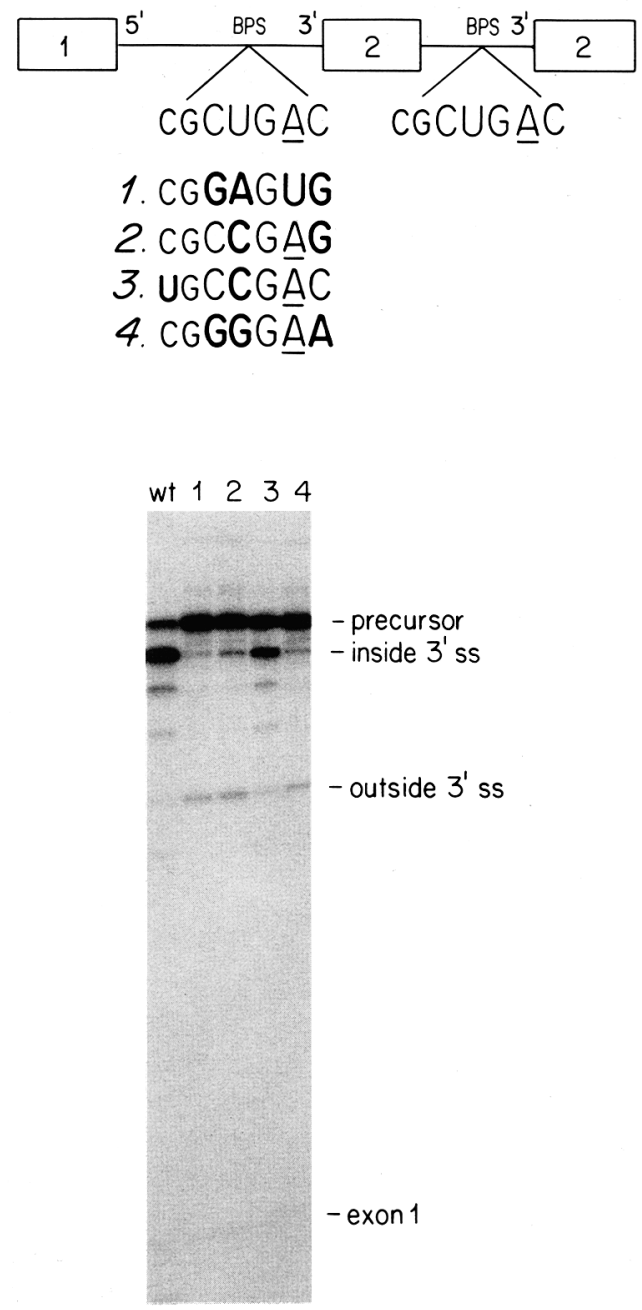

Figure 2. The effect of mutations in the BPS on splicing in vitro. (Top) The structure of the SP6 RNA precursors that were spliced in vitro for $2 \mathrm{hr}$ and then analyzed on a $6 \%$ denaturing polyacrylamide gel. The branch-site adenine is underlined, and mutations in the internal BPS (1-4) are indicated by boldface lettering. The splicing products generated from these precursors are shown in the corresponding gel lanes (1-4). (wt) Normal BPS adjacent to both duplicated $3^{\prime}$ splice sites.

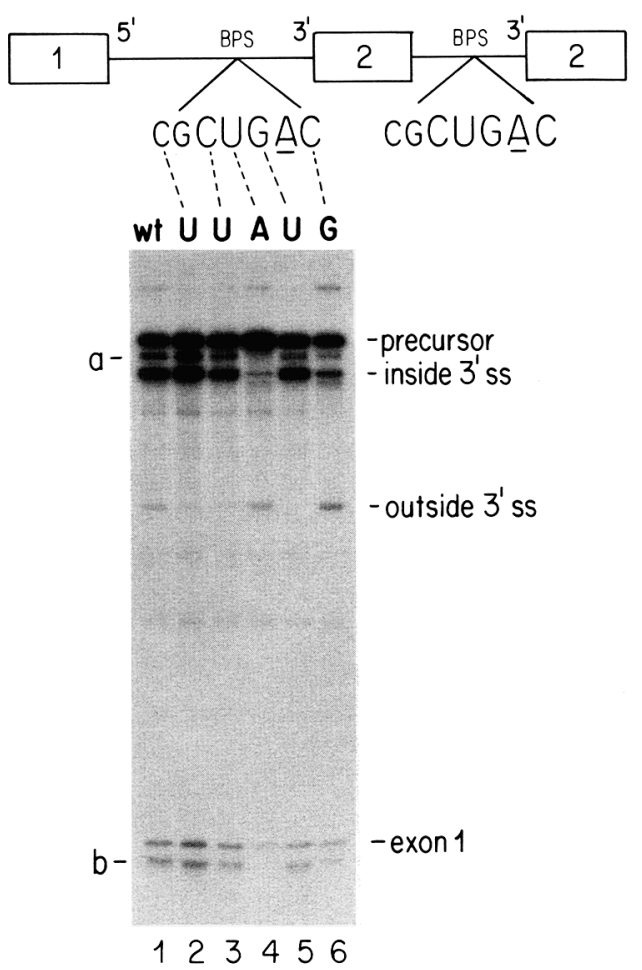

Figure 3. The effect of single-base changes in the BPS on splicing in vitro. (Top) The structure of the SP6 precursors spliced in vitro for $1 \mathrm{hr}$ and then fractionated on a 6\% denaturing polyacrylamide gel. The single-base changes in the BPS adjacent to the internal $3^{\prime}$ splice site are indicated above the corresponding lanes of the gel. (wt) Normal BPS adjacent to both $3^{\prime}$ splice sites. (a) Lariat-exon intermediate derived from use of the internal $3^{\prime}$ splice site; $(b)$ excised lariat intron derived from use of the internal $3^{\prime}$ splice site. The faint bands above the precursor are the lariat introns derived from use of the external $3^{\prime}$ splice site.

of the mutant precursors (Fig. 2, lanes 1, 2, 4) relative to the control (Fig. 2, lane wt). The mutant BPS containing two transitions (Fig. 2, lane 3) was spliced less efficiently than the control precursor but not as poorly as the other mutants. In addition to decreased use of the internal 3' splice site, the use of the external $3^{\prime}$ splice site was increased slightly with all of the mutant precursors compared with the control. We conclude that an adenine nucleotide near the $3^{\prime}$ splice site is not sufficient for normal levels of splicing, and that other nucleotides in the BPS are required.

\section{Single-base changes in the BPS affect splicing}

Examination of several point mutations in the BPS revealed individual nucleotides that affect the efficiency of splicing of precursors lacking the potential cryptic branch sites. The most severe effect on splicing efficiency was observed with a precursor containing a $\mathrm{U} \rightarrow \mathrm{A}$ change at the fourth position of the BPS (Fig. 3; lane 4). In fact, alteration of this nucleotide had as strong an effect on splicing as mutations in the branch-site adenine itself (Fig. 1B, lanes 1,2). Mutation of $C \rightarrow G$ in 
the last position of the BPS (Fig. 3, lane 6) also reduced the use of the internal $3^{\prime}$ splice site, though not as severely as the $\mathrm{U} \rightarrow$ A change. In addition, the use of the external $3^{\prime}$ splice site is increased with the $\mathrm{C} \rightarrow \mathrm{G} \mathrm{mu-}$ tant relative to the control precursor.

In contrast to these down mutations, a $\mathrm{C} \rightarrow \mathrm{U}$ change in the first position of the BPS leads to a small but reproducible increase in the use of the internal $3^{\prime}$ splice site and a decrease in the use of the external site, compared with the control precursor (Fig. 3, lanes 1, 2). Finally, a $\mathrm{G} \rightarrow \mathrm{U}$ change at the weakly conserved position 5 of the BPS (Fig. 3, lane 5) or a transition in the third nucleotide of the BPS (Fig. 3, lane 3) do not significantly affect the use of the internal $3^{\prime}$ splice site but slightly decrease the level of splicing to the external 3' splice site (Fig. 3, lanes $1,3,5)$. The reason for this decrease is not understood.

$B P S$ mutations that inhibit splicing do not prevent spliceosome assembly

In our previous study, we found that inactivation of the internal $3^{\prime}$ splice site by exon mutations leads to a corresponding increase in use of the external $3^{\prime}$ splice site (Reed and Maniatis 1986). In contrast, a similar increase in the use of the external $3^{\prime}$ splice site is not observed with BPS mutations that decrease use of the internal 3' splice site. One possible explanation for the inefficient use of the external $3^{\prime}$ splice site with these precursors is that the BPS mutations do not prevent spliceosome assembly but do prevent $5^{\prime}$ cleavage and lariat formation. If this hypothesis is correct, then a spliceosome would assemble with the internal $3^{\prime}$ splice site because this splice site is preferentially used in the duplication precursors. Stable assembly of this spliceosome would then preclude spliceosome formation and splicing to the external $3^{\prime}$ splice site.

To investigate the effect of BPS mutations on spliceosome assembly, we examined the sedimentation behavior of splicing complexes formed with precursors containing mutations in the BPS but lacking the external duplicated $3^{\prime}$ splice site. The structure of these precursors and the positions of the mutations in the BPS are shown in Figure 4A. Analysis of the splicing products obtained with these precursors revealed that the effects on splicing efficiency observed with the BPS mutations are the same in the presence or absence of the duplicated 3 ' splice sites (cf. Fig. 4B with Figs. 2 and 3).

Density gradient sedimentation analysis of splicing complexes formed with these precursors is shown in Figure 4C. A spliceosome (or $60 \mathrm{~S}$ complex) was formed with precursor 2, which is spliced efficiently (Fig. 4B, lane 2; Fig. 4C, panel 2), as well as with the precursor containing a normal BPS (data not shown). Remarkably, precursors containing BPS mutations that severely inhibit splicing (Fig. 4A, B, lanes 3, 4, 6) also form high levels of 60 S complex (Fig. 4C, panels 3, 6, and data not shown). Although these levels are lower than that observed with the control precursor, a quantitative comparison of the rates of spliceosome assembly with mutant and control precursors is not meaningful, because the spliceosomes assembled on control precursors are transient intermediates. In contrast, spliceosomes assembled on mutant precursors are blocked prior to the first step in the splicing reaction. We conclude that the BPS does not play a central role in spliceosome assembly but is required for efficient cleavage at the $5^{\prime}$ splice site and lariat formation. Thus, BPS mutations appear to uncouple spliceosome assembly and the first step in the splicing reaction.

The $60 \mathrm{~S}$ complexes formed on mutant precursors appear to be stable, as they were observed after $60 \mathrm{~min}$ of incubation in the splicing extract. In contrast, no spliceosomes are detected under comparable conditions with the control precursor, because all of the RNA was spliced by this time point (data not shown). This observation is consistent with the hypothesis that stable complex formation using the internal $3^{\prime}$ splice site results in the relatively inefficient use of the external $3^{\prime}$ splice site with the $3^{\prime}$ duplication precursors containing mutations in the BPS.

\section{The BPS plays a role in $3^{\prime}$ splice-site selection in vivo}

In the experiments described above, we demonstrated that some mutations in the BPS decrease the efficiency of splicing in vitro. To determine whether this sequence element has a role during splicing in vivo, we carried out transient transfection experiments in HeLa cells. The structure of the transcription unit used in these experiments is shown in the schematic diagram in Figure 5. The control plasmid contains the normal BPS adjacent to both duplicated $3^{\prime}$ splice sites, whereas the mutant plasmid contains a $U \rightarrow A$ change at the fourth position of the internal BPS. RNA was prepared $40 \mathrm{hr}$ after transfection, and the splicing products were analyzed by quantitative RNase mapping (Zinn et al. 1983).

As shown in Figure 5 (lane 3), the internal $3^{\prime}$ splice site is used preferentially with the control precursor. In contrast, the external $3^{\prime}$ splice site, adjacent to the normal BPS, is used preferentially with the precursor containing the mutation in the internal BPS (Fig. 5, lane $2)$. Thus, a single-base change in the BPS completely switches the pattern of 3 ' splice-site selection in vivo.

A few differences are apparent between the in vitro (Fig. 3, lane 4) and in vivo (Fig. 5, lane 2) splicing patterns obtained with the precursor containing a mutant BPS. First, high levels of unspliced precursor accumulate in vitro, whereas no unspliced precursor is observed in vivo. Although the reason for this difference is not known, we speculate that, in vivo, the spliceosomes assembled on the internal $3^{\prime}$ splice site are either turned over rapidly or are reassembled using the external $3^{\prime}$ splice site. In contrast, in vitro, these spliceosomes remain stably associated with the pre-mRNA, resulting in its accumulation. Second, the overall level of splicing is decreased significantly with the mutant precursor in vitro, whereas similar amounts of spliced RNA accumulate with both the normal and mutant precursors in vivo. As was documented for the yeast actin intron (Pikielny and Rosbash 1985), splicing may not be the ratelimiting step in the production of stable RNA in vivo. 
A.

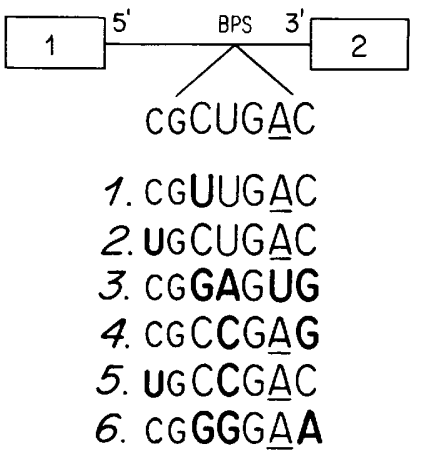

$B$.

Figure 4. The BPS is not required for the assembly of a $60 S$ splicing complex. $(A)$ The structure of the SP6 precursors and the sequence of each mutant BPS $(1-6)$ are shown. The changes relative to the normal BPS are indicated by boldface letters and the branch-site adenine is underlined. $(B)$ The splicing products generated from each of the precursors (after splicing in vitro for 1 $\mathrm{hr}$ ) are indicated in the corresponding gel lanes $(1-6)$. (C) The glycerol gradient profiles of splicing reactions containing precursors 2,3 , or 6 . Precursor 2 was incubated under splicing conditions for $30 \mathrm{~min}$, whereas precursors 3 and 6 were incubated for $1 \mathrm{hr}$. The position of the spliceosome peak (SS) is indicated on the profile for precursor 2. The peak in fractions $5-8$ is the $30 \mathrm{~S}$ peak described previously (Frendewey and Keller 1985 ), and the peak in fractions $10-12$ is ${ }^{32}$ P-labeled nucleotides generated by degradation of RNA during the splicing reaction.

Thus, even mutations that result in significant decreases in splicing efficiency in vitro may not be detected when stable RNA is assayed in vivo.

\section{Discussion}

In this paper we show that a number of mutations in the mammalian BPS result in a substantial decrease in splicing efficiency in vitro. In addition, we show that a single-base change in the BPS alters 3 ' splice-site selection in vivo. Based on these observations and previous studies of the BPS, selection of the branch-site nucleotide appears to be determined not only by the distance from the 3 ' splice site but also by the sequences surrounding the branched nucleotide. Although the BPS does not appear to play a central role in spliceosome as-
C.

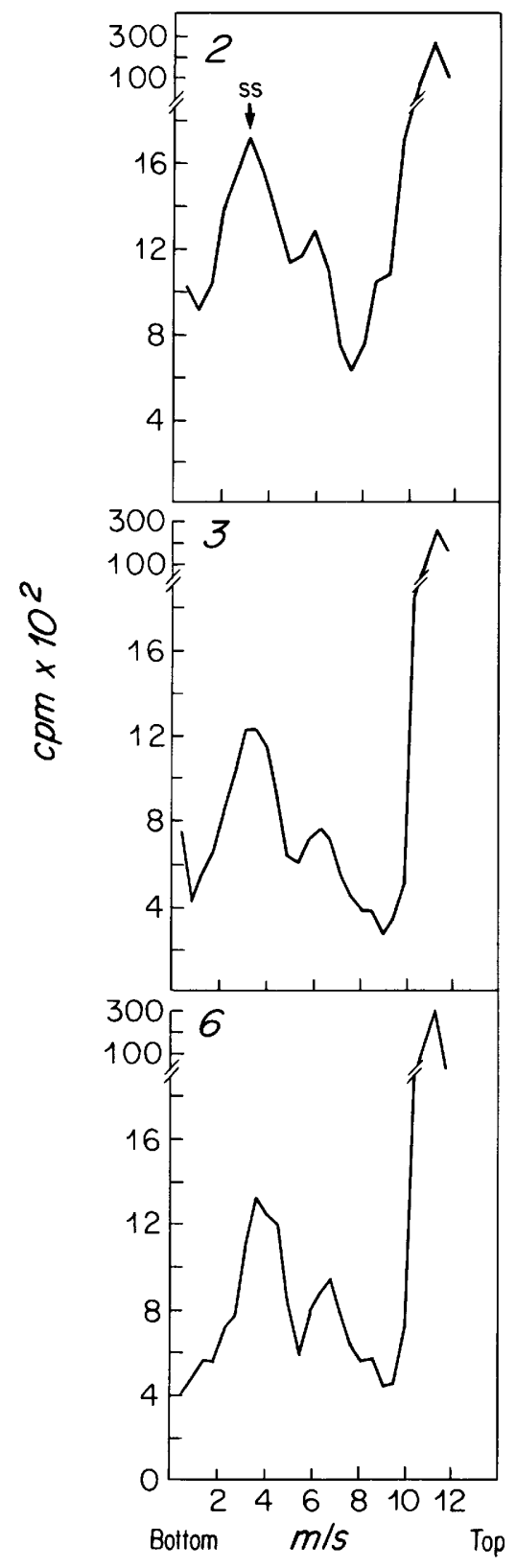

sembly, we show that this sequence element is important in subsequent steps of the splicing reaction.

In earlier studies, deletion of the BPS or alteration of the branch-site adenine decreased the efficiency of splicing in vitro but had no apparent effect on splicing in vivo (Wieringa et al. 1984; Padgett et al. 1985; Ruskin et al. 1985). Two differences may account for this apparent discrepancy with our results. First, in the early studies, the effects of BPS mutations were examined by quantifying the stable mRNA generated from splicing a premRNA containing a single intron. If splicing is not the rate-limiting step in the accumulation of stable RNA, as was shown in yeast (Pikielny and Rosbash 1985), mutations that decrease the efficiency of splicing in vitro may not have an effect in vivo. In contrast, mutations that 


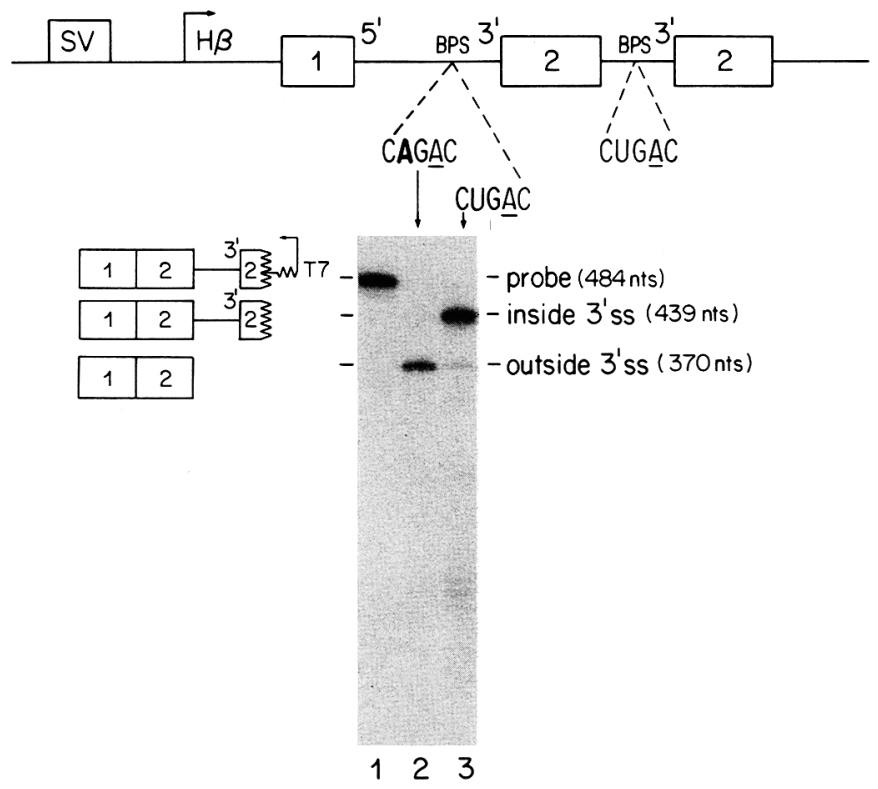

decrease the rate of splicing are readily detected in the cis-competition assay by an alteration in the pattern of 3 ' splice-site selection. Second, the activation of cryptic branch sites decreased the effect of the BPS mutations in the earlier studies, whereas potential cryptic branch sites were eliminated in our analysis.

\section{Comparison of the effects of mutations in the BPS in} yeast and mammals

Mutations in the BPS have similar relative effects on splicing efficiency in mammals and yeast. Substitution of $U$ with $A$ at position 4 of both the mammalian BPS and the yeast UACUAAC box virtually abolishes splicing (Fig. 3, lane 4; Vijayraghavan et al. 1986), whereas a transversion at position 7 is less severe in both (Fig. 3, lane 6; Fouser and Friesen 1986). A transition at position 3 or a transversion at position 5 in the BPS has little effect on splicing, similar to the analogous mutations in the UACUAAC box (Fig. 3, lanes 3, 5; Jacquirer et al. 1985; Fouser and Friesen 1986; Vijayraghavan et al. 1986). Finally, a $\mathrm{C} \rightarrow \mathrm{U}$ change at position 1 of the BPS results in a slight increase in splicing efficiency (Fig. 3, lane 2). Interestingly, this mutation increases the match of the BPS to the yeast UACUAAC box.

The similarities between the effects of mutations in the yeast and mammalian BPSs suggest the possibility that base-pairing between the BPSs and U2 snRNA may be involved in splicing, as in yeast (Parker et al. 1987). This possibility is suggested further by the observation that mammalian U2 snRNP binds to a region of the premRNA that includes the BPS (Black et al. 1985). In addition, the sequence in the yeast U2-like snRNA that base-pairs with the UACUAAC box is conserved in the mammalian U2 snRNA and could potentially base-pair with the BPS (Parker et al. 1987).

Although this hypothesis remains to be tested, it is
Figure 5. A single point mutation in the BPS alters 3 ' splicesite selection in vivo. The structure of the gene that was transiently expressed in HeLa cells is shown. The branch-site adenine nucleotide is underlined, and the mutation in the BPS is indicated by a boldface letter. The SV40 enhancer (SV) was used to obtain expression from the human $\beta$-globin promoter $(\mathrm{H} \beta)$. Total cellular RNA was prepared $40 \mathrm{hr}$ after transfection and then analyzed by RNase mapping. A plasmid containing the human $\beta$-globin gene was cotransfected, and $\alpha$-globin mRNA was mapped as an internal control for transfection efficiency (data not shown). (Lane 1) ${ }^{32} \mathrm{P}$-labeled T7 transcript used as probe for RNase mapping; (lane 2) RNase mapping of RNA prepared from cells transfected with the plasmid bearing the $\mathrm{U} \rightarrow \mathrm{A}$ change in the internal BPS; (lane 3) RNase mapping of RNA prepared from cells transfected with the plasmid bearing two normal BPSs. The structure and sizes of the T7 probe and the RNase-resistant fragments are shown.

striking that the $U \rightarrow$ A change in position 4, which dramatically reduces splicing efficiency in vitro and alters $3^{\prime}$ splice-site selection in vivo would create an A-A mismatch between U2 snRNA and the BPS. In yeast, the effects of the corresponding mutation can be suppressed by a compensatory change in the U2-like snRNA (Parker et al. 1987). As shown in Table 1, the effects of the other BPS mutations are consistent, for the most part, with the hypothesis that U2 snRNA may base-pair with the BPS.

Although mutations in the BPS and UACUAAC box have similar relative effects on splicing efficiency, the roles of these elements in spliceosome assembly in vitro appear to be quite different. We found that mutations in the BPS that essentially abolish splicing still allow spliceosome assembly. In contrast, the analogous mutations in the yeast UACUAAC box prevent spliceosome formation completely (Pikielny et al. 1986; Vijayraghavan et al. 1986). Interestingly, the role of the $3^{\prime}$ splice site also differs between mammals and yeast. Deletion of the $3^{\prime}$ splice site in mammalian introns prevents spliceosome assembly (Frendewey and Keller 1985; Binderief and Green 1986) and 5' cleavage and lariat formation (Reed and Maniatis 1985; Ruskin and Green 1985), whereas the analogous mutation in yeast has little effect on either step (Rymond and Rosbash 1985; Cellini et al. 1986; Fouser and Freisen 1986; Rymond et al. 1987). Thus, both the $3^{\prime}$ splice site and the sequences at the site of lariat formation are involved in more than one step in the splicing reaction, but the relative roles of these sequence elements in some of the steps appear to have changed in yeast and mammals.

During spliceosome assembly, U2 snRNP binds to sequences encompassing the branch site (Black et al. 1985). The $5^{\prime}$ terminus of U2 snRNA is required for this snRNP to bind, whereas internal sequences in U2 snRNA, which are complementary to the BPS (Parker et al. 1987), are not required until a subsequent step (Fren- 
Table 1. Potential base-pairing between U2 snRNA and mutant BPS sequences: correlation with splicing phenotypes

\begin{tabular}{ccccclll}
\hline $3^{\prime}-\mathrm{A}$ & $\mathrm{U}$ & $\mathrm{G}$ & $\mathrm{A}$ & $\mathrm{U}$ & & $\mathrm{G}-5^{\prime}$ & $\mathrm{U} 2$ snRNA \\
& $\mathrm{I}$ & $\mathrm{I}$ & $\downarrow$ & $\mathrm{I}$ & & I & \\
$5^{\prime}-\mathrm{C}$ & $\mathrm{G}$ & $\mathrm{C}$ & $\mathrm{U}$ & $\mathrm{G}$ & $\mathrm{A}$ & $\mathrm{C}-3^{\prime}$ & mammalian BPS \\
$\downarrow$ & & $\downarrow$ & $\downarrow$ & $\downarrow$ & & $\downarrow$ & \\
$\mathrm{U}$ & & $\mathrm{U}$ & $\mathrm{A}$ & $\mathrm{U}$ & - & $\mathrm{G}$ & BPS point mutations \\
+ & & 0 & - & - & - & $\begin{array}{l}\text { potential base pairing } \\
\text { splicing phenotypes }^{\mathrm{b}}\end{array}$ \\
$\uparrow$ & & $\mathrm{wt}$ & $\downarrow \downarrow$ & $\mathrm{wt}$ & $\downarrow$ & $\downarrow$
\end{tabular}

a Potential base-pairing between U2 snRNA and BPS mutants. $(+\mid$ The $C \rightarrow U$ mutation in the BPS creates a U-A bp with U2 snRNA. (0) Base-pairing is unaffected by the $C \rightarrow U$ change because a G-U bp is created. (-) The $U \rightarrow A, G \rightarrow U$, and $C \rightarrow G$ changes disrupt base-pairing with U2 snRNA.

b Effect of BPS mutations on splicing efficiency. (wt) Normal; $(\uparrow)$ slightly increased; $(\downarrow)$ decreased; $(\downarrow \downarrow)$ severely decreased.

dewey et al. 1987). These observations led to the proposal that U2 snRNP binds to the BPS region, based primarily on its distance from the $3^{\prime}$ splice site. This distance may be established by U2AF, a factor that binds to the $3^{\prime}$ splice site and appears to be required for U2 snRNP binding at the branch site (Ruskin et al. 1988). The sequence of the BPS is then required for the splicing reaction, possibly by base-pairing with U2 snRNP (Frendewey et al. 1987; Steitz et al. 1988). Our data are consistent with this model because we observe spliceosome assembly in the absence of an intact BPS but require this sequence element for cleavage at the $5^{\prime}$ splice site and lariat formation.

\section{Splice-site selection in mammalian pre-mRNAs}

The sequences necessary for splicing in higher eukaryotes have been defined, for the most part, using premRNAs containing a single small intron. The specific sequence elements required for accurate splicing of such introns are located at the $5^{\prime}$ and $3^{\prime}$ splice junctions. However, most naturally occurring pre-mRNAs in higher eukaryotes are highly complex, containing multiple introns, which range in size from 65 to $100,000 \mathrm{nu}-$ cleotides. In addition, many pre-mRNAs are alternatively spliced. Thus, in contrast to the single small intron found in yeast pre-mRNAs, or to the prototype pre-mRNAs used to study splicing in higher eukaryotes, additional mechanisms are required for accurate splicesite selection in naturally occurring metazoan premRNAs.

Although these mechanisms are not understood, accurate splice-site selection may be achieved through a complex and delicate balance between the splicing efficiencies of each intron in the pre-mRNA. These efficiencies, in turn, could be modulated by a number of parameters, including sequence variability in the recognition elements at the $5^{\prime}$ and $3^{\prime}$ splice sites and at the BPS. The use of multiple branch sites with varying degrees of homology to the BPS or the use of C, U, or G residues as branch-site nucleotides may also affect splicing efficiencies and the selection of alternative splice sites (Hornig et al. 1986; Freyer et al. 1987; Noble et al. 1987; J.C.S. Noble, C. Prives, and J.L. Manley, pers. comm.). Finally, the flanking exon sequences and the proximity of splice sites to one another play a role in determining splicing efficiencies (Reed and Maniatis 1986). Each of these parameters is a potential target for regulation of alternatively spliced pre-mRNAs.

\section{Experimental procedures}

\section{BPS plasmid constructions}

The SP64 plasmids used in the cis-competition experiments were based on the plasmid 3'D-205 described in Reed and Maniatis (1986). The derivative of $3^{\prime} D-205$ that contains an $A \rightarrow G$ base change in the branch-site adenine (in the presence of cryptic branch sites) was constructed from the human $\beta$-globin $A \rightarrow G$ substitution mutant (Ruskin et al. 1985; a gift from M. Green).

To construct the plasmids containing BPS mutations (see below for mutagenesis scheme), an XhoI linker was first inserted into plasmid $H \beta-\Delta B a m$ (Reed and Maniatis 1986) at the $M b o I I$ site located at position +77 , relative to the $5^{\prime}$ end of the intron. Complementary oligodeoxynucleotides $(\sim 70 \mathrm{bp})$, containing IVS 1 sequences from position +77 to +143 relative to the $5^{\prime}$ splice site, were synthesized with an XhoI site at the $5^{\prime}$ end and an $A c c$ site at the $3^{\prime}$ end. The $70-\mathrm{bp}$ oligodeoxynucleotides were then inserted into $\mathrm{H} \beta-\Delta \mathrm{Bam}$ at the $X h \mathrm{I}$ and $A c c \mathrm{I}$ sites (located at +143 ). Potential cryptic branchpoint sites were eliminated from the 3 ' region of the intron by substituting the As at positions 84 and 90 with $G$ residues and the As at positions 86, 107, and 115 with Ts. The sequences of these SP64 plasmids, designated $\mathrm{H} \beta$-BPS series, were determined by chain termination sequencing as described previously (Sanger et al. 1977).

The control plasmid, containing tandemly duplicated $3^{\prime}$ splice sites (wt) with normal BPSs (CGCTGAC) adjacent to both 3 ' splice sites, was subsequently constructed by inserting the XhoI (blunt-ended with Klenow polymerase), EcoRI fragment (sites +77 to +225 ) from plasmid H $\beta$-BPS (containing the normal BPS) into the SmaI-EcoRI sites $(+500$ to +510$)$ of the same plasmid. The plasmids containing mutations in the BPS adjacent to the internal duplicated $3^{\prime}$ splice site and the normal BPS adjacent to the external $3^{\prime}$ splice site were constructed in the same manner, except that the XhoI-EcoRI fragment was derived from H $\beta$-BPS plasmids containing mutations in the BPS.

\section{Mutagenesis of BPS}

Mutations in the seven nucleotides comprising the BPS (CGCTGAC) were created using the method of Hutchison et al. 
(1986). During the synthesis of each strand of the $\sim 70$-bp duplex DNA containing the $3^{\prime}$ end of $\beta$-globin IVS 1 , reservoirs containing $79 \%$ of the correct nucleotide and $7 \%$ each of the other three were substituted at the time of synthesis of the seven BPS nucleotides. Complementary oligonucleotides synthesized in this manner were annealed and inserted into the plasmid described above. Sequence analysis of plasmids from randomly picked colonies revealed from one to four base substitions in the BPS.

\section{Pre-mRNA synthesis and in vitro splicing}

The SP6 precursors containing one $3^{\prime}$ splice site or tandemly duplicated $3^{\prime}$ sites were linearized with BamHI or EcoRI, respectively. Pre-mRNA synthesis using SP6 (or T7) polymerase was performed as described in Krainer et al. (1984), with modifications described in Reed et al. (1988). In vitro splicing reactions were carried out for 1 or $2 \mathrm{hr}$ (as indicated) and contained 5-10 ng of each ${ }^{32}$ P-labeled RNA precursor (Krainer et al. 1984).

\section{Density gradient sedimentation}

Splicing reactions $(100 \mu \mathrm{l})$ were sedimented on $10-30 \%$ glycerol gradients (11 ml), as described (Grabowski et al. 1985), with modifications (Abmayr et al. 1988).

\section{Transient transfection and RNase mapping}

The expression plasmids used for transfection into HeLa cells were constructed by ligating the following three fragments together: an NcoI-EcoRI fragment (from plasmid $\pi S V H S \beta \Delta 128 \mathrm{E}$; Little et al. 1983; Treisman et al. 1983) that contains the SV40 enhancer and the human $\beta$-globin promoter, an NcoI-SmaI fragment containing the sequences encoding the $3^{\prime}$ splice-site duplication precursors, and a Bg/II (blunt-ended with Klenow polymerase)-EcoRI fragment carrying the rabbit $\beta$-globin polyadenylation signals and SP64 vector sequences (from SP64R $\beta$ G; L. Whittemore, unpubl.). The resultant plasmids contained sequences encoding the control 3' splice-site duplication precursor or the $\mathrm{T} \rightarrow \mathrm{A}$ mutation in the internal BPS /see above and Fig. 31.

Transient expression assays, RNA preparations, and RNA analyses were performed as described previously (Treisman et al. 1983; Zinn et al. 1983). The plasmid used as a probe for RNase mapping was constructed by ligating a HindIII-AvaII $(+1$ to +175$)$ fragment from a human $\beta$-globin cDNA clone, and $A v a \mathrm{II}-A c c \mathrm{I}$ (blunted with Klenow polymerase) fragment from the control H $\beta$-BPS plasmid clone /containing a portion of the internal exon 2 sequences and the duplicated region of IVS 1 and a portion of the duplicated region of exon 2), into the SmaI-HindIII sites of SP72 (gift from D. Melton). For in vitro transcription using $\mathrm{T} 7$ polymerase, the plasmid was digested with HindIII.

\section{Acknowledgments}

We thank Gene Brown (Genetics Institute) for synthesizing the oligonucleotides used for mutagenesis. We gratefully acknowledge Lisa Whittemore for valuable advice on the in vivo experiments and thank Susan Abmayr, Chen-Ming Fan, Kevin Jarrell, Xiang Dang Fu, and R-J. Lin for useful discussions and comments on the manuscript. R.R. is a Lucille P. Markey Scholar. This work was supported, in part, by a grant from the Lucille $P$. Markey Charitable Trust and by a grant to T.M. from the National Institutes of Health (HL-27898).

\section{Note added in proof}

Similar observations regarding the role of the BPS in pre-mRNA splicing were made independently by Y. Zhuang, A. Goldstein, and A.M. Weiner (pers. comm.).

\section{References}

Abmayr, S.M., R. Reed, and T. Maniatis. 1988. Identification and functional analysis of a mammalian splicing complex. Proc. Natl. Acad. Sci. (in press).

Bindereif, A. and M.R. Green. 1986. Ribonucleoprotein complex formation during pre-mRNA splicing in vitro. Mol. Cell. Biol. 6: 2582-2592.

Black, D.L., B. Chabot, and J.A. Steitz. 1985. U2 as well as U1 small nuclear ribonucleoproteins are involved in pre-messenger RNA splicing. Cell 42: 737-750.

Cellini, A., E. Felder, and J.J. Rossi. 1986. Yeast pre-messenger RNA splicing efficiency depends on critical spacing requirements between branch point and 3 ' splice site. EMBO $J$. 5: $1023-1030$.

Fouser, L.A. and J.D. Friesen. 1986. Mutations in a yeast intron demonstrate the importance of specific conserved nucleotides for the two stages of nuclear mRNA splicing. Cell 45: 81-93.

Frendewey, D. and W. Keller. 1985. The stepwise assembly of a pre-mRNA splicing complex requires $U$ snRNPs and specific intron sequences. Cell 42: 355-367.

Frendewey, D., A. Kramer, and W. Keller. 1987. Different small nuclear ribonucleoprotein particles are involved in different steps of splicing complex formation. Cold Spring Harbor Symp. Quant. Biol. (in press).

Freyer, G., J. Arenas, K.K. Perkins, H.M. Furneaux, L. Pick, B. Young, R.J. Roberts, and J. Hurwitz. 1987. In vitro formation of a lariat structure containing a $\mathrm{G}^{2}{ }^{\prime} 5^{\prime} \mathrm{G}$ linkage. I. Biol. Chem. 262: 4267-4273.

Grabowski, P.J., S.R. Seiler, and P.A. Sharp. 1985. A multicomponent complex is involved in the splicing of messenger RNA precursors. Cell 42: 345-353.

Green, M.R. 1986. Pre-mRNA splicing. Annu. Rev. Genet. 20: $671-708$.

Hartmuth, K. and A. Barta. 1988. Unusual branch point selection in processing of human growth hormone pre-mRNA. Mol. Cell. Biol. 8: 2011-2020.

Hornig, H., M. Aebi, and C. Weissman. 1986. Effect of mutations at the lariat branch acceptor site on $\beta$-globin premRNA splicing in vitro. Nature 324: 589-591.

Hutchison, C.A.., III, S.K. Nordeen, K. Vogt, and M.H. Edgell. 1986. A complete library of point substitution mutations in the glucocorticoid response element of mouse mammary tumor virus. Proc. Natl. Acad. Sci. 83: 710-714.

Jacquier, A., J.R. Rodriguez, and M. Rosbash. 1985. A quantitative analysis of the effects of $5^{\prime}$ junction and TACTAAC box mutants and mutant combinations on yeast mRNA splicing. Cell 43: 423-430.

Keller, E.B. and W.A. Noon. 1984. Intron splicing: A conserved internal signal in introns of animal pre-mRNAs. Proc. Natl. Acad. Sci. 81: 7417-7420.

Krainer, A.R. and T. Maniatis. 1988. RNA splicing. In Frontiers in Molecular transcription and splicing (ed. B.D. Hames and D.M. Glover), Biology, IRL Press, Oxford and Washington, D.C., pp. 131-206.

Krainer, A.R., T. Maniatis, B. Ruskin, and M.R. Green. 1984 Normal and mutant human $\beta$-globin pre-mRNAs are faithfully and efficiently spliced in vitro. Cell 42: 725-736. 
Little, P.F.R., R. Treisman, L. Bierut, B. Seed, and T. Maniatis. 1983. Plasmid vectors for the rapid isolation and transcriptional analysis of human $\beta$-globin gene alleles. Mol. Biol. Med. 1: 473-488.

Noble, J.C.S., Z.-Q. Pan, C. Prives, and J.L. Manley. 1987. Splicing of SV40 early pre-mRNA to large T and small $t$ mRNAs utilizes different patterns of lariat branch sites. Cell 50: 227-236.

Padgett, R.A., P.J. Grabowski, M.M. Konarska, S. Seiler, and P.A. Sharp. 1986. Splicing of messenger RNA precursors. Annu. Rev. Biochem. 55: 1119-1150.

Padgett, R.A., M.M. Konarska, M. Aebi, H. Hornig, C. Weissmann, and P.A. Sharp. 1985. Nonconsensus branch-site sequences in the in vitro splicing of transcripts of mutant rabbit $\beta$-globin genes. Proc. Natl. Acad. Sci. 82: 8349-8353.

Parker, R., P.G. Siliciano, and C. Guthrie. 1987. Recognition of the TACTAAC box during mRNA splicing in yeast involves base pairing to the U2-like snRNA. Cell 49: 229-239.

Pikielny, C.W. and M. Rosbash. 1985. mRNA splicing efficiency in yeast and the contribution of nonconserved sequences. Cell 41: 119-126.

Pikielny, C.W., B.C. Rymond, and M. Rosbash. 1986. Electrophoresis of ribonucleoproteins reveals an ordered assembly pathway of yeast splicing complexes. Nature 324: 341-345.

Rautmann, G. and R. Breathnach. 1985. A role for branchpoints in splicing in vivo. Nature 315: 430-432.

Rautmann, G., H.W.D. Matthes, M.J. Gait, and R. Breathnach. 1984. Synthetic donor and acceptor splice sites function in an RNA polymerase B (II) transcription unit. EMBO $/$. 3: 2021-2028.

Reed, R. and T. Maniatis. 1985. Intron sequences involved in lariat formation during pre-mRNA splicing. Cell 41: 95105.

1986. A role for exon sequences and splice-site proximity in splice-site selection. Cell 46: 681-690.

Reed, R., J. Griffith, and T. Maniatis. 1988. Purification and visualization of native spliceosomes. Cell 53: 949-961.

Ruskin, B. and M.R. Green. 1985. Role of the 3' splice site consensus sequence in mammalian pre-mRNA splicing. Nature 317: 732-734.

Ruskin, B., J.M. Greene, and M.R. Green. 1985. Cryptic branch point activation allows accurate in vitro splicing of human $\beta$-globin intron mutants. Cell 41: 833-844.

Ruskin, B., P.D. Zamore, and M.R. Green. 1988. A factor, $\mathrm{U} 2 \mathrm{AF}$, is required for U2 snRNP binding and splicing complex assembly. Cell 52: 207-219.

Ruskin, B., A.R. Krainer, T. Maniatis, and M.R. Green. 1984. Excision of an intact intron as a novel lariat structure during pre-mRNA splicing in vitro. Cell 38: $317-331$.

Rymond, B.C. and M. Rosbash. 1985. Cleavage of 5' splice site and lariat formation are independent of $3^{\prime}$ splice site in yeast mRNA splicing. Nature 317: 735-737.

Rymond, B.C., D.D. Torrey, and M. Rosbash. 1987. A novel role for the $3^{\prime}$ region of introns in pre-mRNA splicing of Saccharomyces cerevisiae. Genes Dev. 1: 238-246.

Sanger, F., S. Nicklen, and A. Coulson. 1977. DNA sequencing with chain-terminating inhibitors. Proc. Natl. Acad. Sci. 74: 5463-5467.

Steitz, J.A., D.L. Black, V. Gerke, K.A. Parker, A. Kramer, D. Frendewey, and W. Keller. 1988. Functions of the abundant U-snRNPs. In Structure and function of major and minor SNURPS (ed. M. Birnsteil), Springer-Verlag, New York (in press).

Treisman, R., S. Orkin, and T. Maniatis. 1983. Specific transcription and RNA splicing defects in five cloned $\beta$-thalassemia genes. Nature 302: 591-596.
Vijayraghavan, U., R. Parker, J. Tamm, Y. Iimura, J. Rossi, J. Abelson, and C. Guthrie. 1986. Mutations in conserved intron sequences affect multiple steps in the yeast splicing pathways, particularly assembly of the spliceosome. $E M B O$ I. 7: 1683-1695.

Wieringa, B., E. Hofer, and C. Weissman. 1984. A minimal intron length but no specific internal sequence is required for splicing the large rabbit $\beta$-globin intron. Cell 37: 915-925.

Zeitlin, S. and A. Efstratiadis. 1984. In vivo splicing products of the rabbit $\beta$-globin pre-mRNA. Cell 39: 589-602.

Zinn, K., D. DiMaio, and T. Maniatis. 1983. Identification of two distinct regulatory regions adjacent to the human $\beta$-interferon gene. Cell 34: 865-879. 


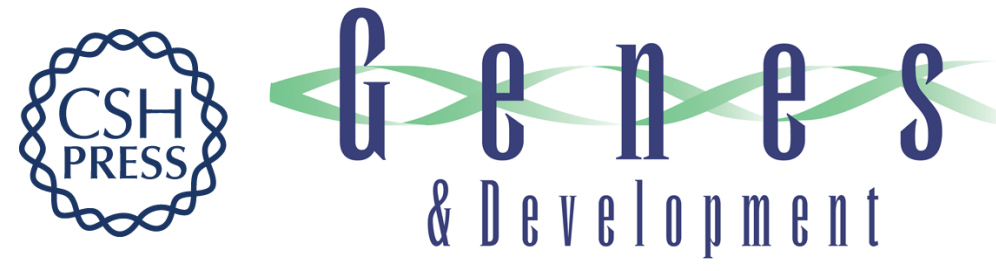

\section{The role of the mammalian branchpoint sequence in pre-mRNA splicing.}

R Reed and T Maniatis

\section{Genes Dev. 1988, 2:}

Access the most recent version at doi:10.1101/gad.2.10.1268

References This article cites 38 articles, 8 of which can be accessed free at:

http://genesdev.cshlp.org/content/2/10/1268.full.html\#ref-list-1

License

Email Alerting

Service

Receive free email alerts when new articles cite this article - sign up in the box at the top right corner of the article or click here.

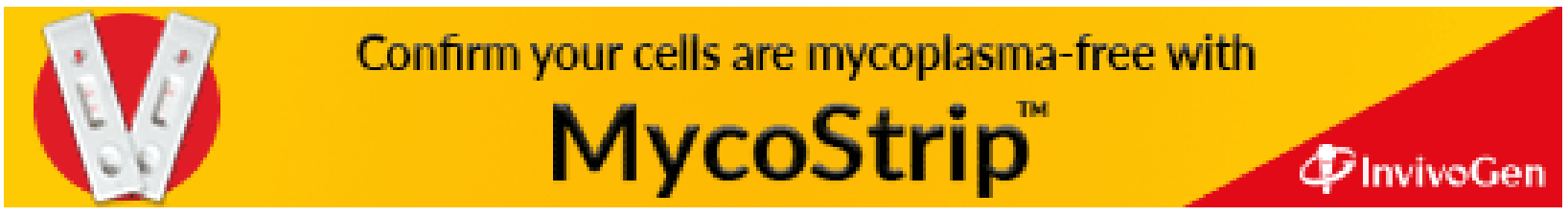

